Bull. Austral. Math. Soc.

VoL. 55 (1997) [7-18]

\title{
BRAID REPRESENTATIONS OF PERIODIC LINKS
}

\author{
Sang Youl Lee and Chan-Young Park
}

In this paper, we study periodic braid representations of periodic links. It is shown that no 2-bridge non-fibred knot has a periodic braid representation and conditions under which periodic links are fibred are given. We give a construction of all periodic links over a fixed factor link.

\section{INTRODUCTION}

An oriented link $L$ in $S^{3}$ is called an $n$-periodic link $(n>1)$ if there is an orientation preserving homeomorphism $\phi:\left(S^{3}, L\right) \rightarrow\left(S^{3}, L\right)$ such that $\phi$ is of order $n$ and the fixed point set $F$ of $\phi$ is a 1-sphere in $S^{3}$ disjoint from $L$. The homeomorphism $\phi$ is called an $n$-periodic homeomorphism for $L$. It is known that $F$ is unknotted, the quotient map $p: S^{3} \rightarrow S^{3} / \phi$ is an $n$-fold cyclic branched covering space branched over $p(F)=F_{*}$, and $p(L)=L_{*}$ is also an oriented link in $S^{3} / \phi \cong S^{3}$, which is called the factor link of $L$.

Let $B_{m}$ be Artin's braid group on $m$ strings. A link $L$ in $S^{3}$ is said to have an $n$-periodic braid representation $(n>1)$ if it is ambient isotopic to the closure of a braid $\alpha^{n}$ for some braid $\alpha$. For a braid $\alpha \in B_{m}$, the closure of the braid $\alpha^{n}$ for $n>1$ is an $n$-periodic link whose factor link is ambient isotopic to the closure of $\alpha$. A periodic link may or may not have a periodic braid representation. In this paper we give some properties of periodic links in $S^{3}$ which have periodic braid representations.

This paper is organised as follows. In section 2 we give some preliminary results. In section 3 we show that if an $n$-periodic link $L$ in $S^{3}$ has an $n$-periodic braid representation, then $L$ is a fibred link or the factor link of $L$ is not the trivial knot. In particular, let $K$ be an $n$-periodic knot of genus $g$ with either $g<n$ or $n=g=2$ or 3 and let $\phi$ be the $n$-periodic homeomorphism for $K$. If $M$ is a $\phi$-invariant Seifert surface for $K$ with genus $g$ such that the boundary of $M$ is an $n$-periodic closed braid with the fixed point set $F$ of $\phi$ as its braid axis, then $K$ is a fibred knot. In section 4 we construct periodic links over a fixed factor link by slightly modifying Bae and Park's construction [2]. This may give many examples of periodic links which have periodic braid representations.

Received 30th January, 1996

This research was supported in part by the Basic Science Research Institute program, Ministry of Education, 1995, Project No. BSRI 95-1409 and by TGRC-KOSEF.

Copyright Clearance Centre, Inc. Serial-fee code: 0004-9729/97 \$A2.00+0.00. 


\section{Preliminaries}

Let $B_{m}$ be Artin's braid group on $m$ strings with the standard generators $\sigma_{1}, \sigma_{2}$, $\cdots, \sigma_{m-1}$, that is,

$$
\begin{aligned}
B_{m}=\left\langle\sigma_{1}, \sigma_{2}, \cdots, \sigma_{m-1}\right| \sigma_{i} \sigma_{i+1} \sigma_{i}=\sigma_{i+1} \sigma_{i} \sigma_{i+1}(1 \leqslant i \leqslant m-2) \\
\left.\sigma_{i} \sigma_{j}=\sigma_{j} \sigma_{i}(|i-j| \geqslant 2)\right\rangle .
\end{aligned}
$$

As usual, for a braid $\beta \in B_{m}$, the closed braid or the closure of $\beta$, denoted by $\beta^{\wedge}$, with an axis $A$ is defined as in the following Figure 2.1 .

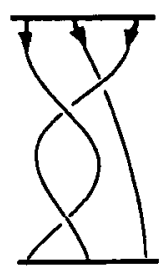

B

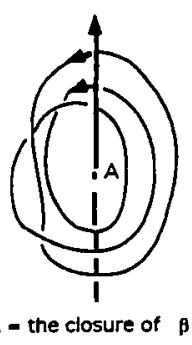

pn - the closure of

Figure 2.1

We give an orientation on $\beta^{\wedge}$ by taking the orientation on each string of $\beta$ from the top to the bottom and then give the orientation on the axis $A$ so that when a point moves along the closed braid in the positive direction, it winds around the axis in the right hand direction. In [1], Alexander showed that every oriented link is ambient isotopic to the closure of some braid.

A Markov move of type $I$ is changing $\beta \in B_{m}$ to $\alpha \beta \alpha^{-1} \in B_{m}$ for any $\alpha \in B_{m}$ and a Markov move of type $I I$ is changing $\beta \in B_{m}$ to $\beta \sigma_{m}^{ \pm 1} \in B_{m+1}$, or the inverse of this operation. Markov's theorem says that two braids $\beta_{1} \in B_{m}$ and $\beta_{2} \in B_{n}$ have ambient isotopic closures if and only if $\beta_{1}$ is Markov equivalent to $\beta_{2}$, that is, there is a finite sequence of Markov moves, which takes $\beta_{1}$ to $\beta_{2}$ [3].

The braid index $b(L)$ of an oriented link $L$ is defined to be the minimum number of strings needed to represent $L$ as a closed braid. A braid representation $\beta$ of an oriented link $L$ is said to be minimal if the number of strings in $\beta$ is equal to the braid index $b(L)$.

Given a link $L$ in $S^{3}$, a Seifert surface for $L$ is a compact connected orientable surface embedded in $S^{3}$ such that the boundary of the surface is the link $L$. The genus of a link in $S^{3}$ is defined to be the least genus of all its Seifert surfaces.

A link $L$ in $S^{3}$ is fibred if the complement $S^{3}-L$ is the total space of a fibre bundle over $S^{1}$ whose fibre is the interior of a Seifert surface for $L$. 
An alternating knot $K$ in $S^{3}$ is fibred if and only if the Alexander polynomial $\Delta_{K}(t)$ of $K$ is monic, that is, the first (and last) nonzero coefficients of $\Delta_{K}(t)$ are \pm 1 $[\mathbf{1 0}, \mathbf{1 1}]$.

\section{Periodic braid Representations of PERIOdic LiNKS}

In this section, all knots or links are oriented unless stated otherwise.

DEFINITION 3.1: A link $L$ in $S^{3}$ is said to have an $n$-periodic braid representation $(n>1)$ if it is ambient isotopic to the closure of a braid of the form $\alpha^{n}$ for some braid $\alpha$.

It is obvious that non-periodic links can not have any periodic braid representation. Let $\beta$ be a braid representation of an $n$-periodic $\operatorname{link} L$. Then $L$ has an $n$-periodic braid representation if and only if $\beta$ is Markov equivalent to some braid of the form $\alpha^{n}$.

In [9], Murasugi verified that any period of the torus knot $K_{m, n}$ of type $(m, n)$ is a divisor of $m$ or $n$. Note that $K_{m, n}$ is ambient isotopic to the closure of $\left[\left(\sigma_{1} \sigma_{2} \cdots \sigma_{n-1}\right)^{m / d}\right]^{d}$ or $\left[\left(\sigma_{1} \sigma_{2} \cdots \sigma_{m-1}\right)^{n / d}\right]^{d}$, where $d$ is a divisor of $m$ or $n$ respectively. Hence the torus knot $K_{m, n}$ has a $d$-periodic braid representation. Furthermore, the torus knot $K_{m, n}$ is a fibred knot. In fact, there are many non-periodic fibred knots. For example, the knot $8_{10}$ is the first such knot in Rolfsen's table [11]. For, $8_{10}$ has no period [7] and the Alexander polynomial of the knot $8_{10}$ is $\Delta_{8_{10}}(t)=-1+3 t-6 t^{2}+7 t^{3}-6 t^{4}+3 t^{5}-t^{6}$. Since it is an alternating knot with monic Alexander polynomial, it is a fibred knot [10]. On the other hand the knot $5_{2}$ is a non-fibred knot with period 2. For, it is an alternating knot of period 2 and the Alexander polynomial of $5_{2}$ is $\Delta_{5_{2}}(t)=-2+3 t-2 t^{2}$ which is not monic.

LEMMA 3.2. Let $\beta$ be a braid representing the trivial knot. Then the closure of the braid $\beta^{n}(n>1)$ is an $n$-periodic fibred link in $S^{3}$.

Proof: Let $\beta$ be a braid representing the trivial knot and let $K=\beta^{\wedge}$. Take the $z$-axis in $\mathbb{R}^{3}$ as a braid axis of the closed braid $K$. We consider $S^{3}$ as $\mathbb{R}^{3} \cup\{\infty\}$ and let $J=$ the $z$-axis $\cup\{\infty\}$, the trivial knot in $S^{3}$. Let $p: S^{3} \rightarrow S^{3}$ be the standard $n$-fold cyclic branched covering space branched along $J$. Then $p^{-1}(K)$ is an $n$-periodic link in $S^{3}$, which is ambient isotopic to the closed braid $K^{\prime}=\left[\beta^{n}\right]^{\wedge}$. Now since $K$ is the trivial knot and hence fibred, the complement $S^{3}-K$ fibres over $S^{1}$ with fibre $D_{t}^{\circ}, t \in S^{1}$, the interior of the 2-disc $D^{2}$ in $S^{3}$ such that the boundary of $D^{2}$ is the trivial knot $K$. Note that $J$ intersects each fibre $D_{t}^{\circ}\left(t \in S^{1}\right)$ transversally in $m$ points, where $m$ is the absolute value of the linking number between $J$ and $K$. Let $M_{t}=p^{-1}\left(\bar{D}_{t}\right)$ for each $t \in S^{1}$, where $\bar{D}_{t}$ denotes the closure of the fibre $D_{t}^{\circ}$. Then the boundary of $M_{t}$ is the link $K^{\prime}$ and $S^{3}-K^{\prime}$ is a locally trivial bundle over $S^{1}$ by virtue of the homotopy 
lifting property of the covering space $p: S^{3}-\left\{K^{\prime} \cup p^{-1}(J)\right\} \rightarrow S^{3}-\{K \cup J\}$. Hence $S^{3}-K^{\prime}$ fibres over $S^{1}$ with fibre $M_{1}^{\circ}=p^{-1}\left(D_{1}^{\circ}\right)\left(1 \in S^{1}\right)$, the interior of a Seifert surface for $K^{\prime}$.

Theorem 3.3. Let $L$ be an $n$-periodic link in $S^{3}$. If $L$ has an $n$-periodic braid representation, then $L$ is a fibred link or the factor link of $L$ is not the trivial knot.

PROOF: Let $\beta^{n}$ be an $n$-periodic braid representation of $L$ and suppose that the factor link of $L$ is the trivial knot. Since the closure $\beta^{\wedge}$ of the braid $\beta$ is the factor link of $L$, it is the trivial knot. Thus by Lemma 3.2, $L$ is a fibred link in $S^{3}$.

COROllary 3.4. No non-fibred 2-bridge knot has a periodic braid representation.

Proof: First we note that the 2-bridge knots which are not torus knots have only period 2 [6], and their factor knots are the trivial knot. Thus it is easy to deduce the assertion from Theorem 3.3.

It should be noted that Corollary 3.4 is not true for a 2-bridge fibred knot. For example, the figure eight knot $4_{1}$ is a 2-bridge fibred knot of only period 2 and $4_{1}$ has a 2-periodic minimal braid representation: $4_{1}=\left[\left(\sigma_{2} \sigma_{1}^{-1}\right)^{2}\right]^{\wedge}$.

In [4], Edmonds proved that a periodic knot of genus $g$ has a Seifert surface with genus $g$ which is invariant under the periodic homeomorphism. Notice that the torus knot $K_{m, n}$ has a $d$-periodic braid representation such that the Seifert surface obtained from the closed $d$-periodic braid by applying Seifert's algorithm is of minimal genus and is invariant under the periodic homeomorphism.

The following Lemma 3.5 is an immediate consequence of the Riemann-Hurwitz Formula, in the proof of Corollary 5 in [4], which asserts that if $K$ is an $n$-periodic knot of genus $g$, then $n \leqslant 2 g+1$.

Lemma 3.5. Let $K$ be an n-periodic knot of genus $g$. Let $M$ be a Seifert surface for $K$ with genus $g$ which is invariant under the $n$-periodic homeomorphism $\phi$ for $K$ and let $g_{*}$ be the genus of the quotient $M / \phi$. Then

$$
g=n g_{*}+\frac{(n-1)(m-1)}{2}
$$

where $m$ is the number of intersection points of $M$ with the fixed point set $F$ of $\phi$ transversally.

Using Lemma 3.5, we now obtain the following:

THEOREM 3.6. Let $K$ be an n-periodic knot of genus $g$ and let $\phi$ be the $n$ periodic homeomorphism for $K$. Let $M$ be a $\phi$-invariant Seifert surface for $K$ with genus $g$. Assume that either $g<n$ or $n=g=2$ or 3 . If the boundary of $M$ is an 
$n$-periodic closed braid with the fixed point set $F$ of $\phi$ as its braid axis, then $K$ is a fibred knot.

Proof: Suppose that $M$ is a $\phi$-invariant Seifert surface for $K$ with genus $g$ such that the boundary of $M$ is an $n$-periodic closed braid $\left[\alpha^{n}\right]^{\wedge}$, for some braid $\alpha$, with $F$ as its braid axis representing the knot $K$. We may assume that $M$ intersects with $F$ transversally. Then the quotient map $p: M \rightarrow M / \phi$ is the $n$-fold cyclic branched covering branched over $m$ points, where $m$ is the number of intersection points of $M$ with $F$. We may assume that the boundary of $M / \phi$ is the closure $\alpha^{\wedge}\left(=p\left(\left[\alpha^{n}\right]^{\wedge}\right)\right)$ of the braid $\alpha$. By Lemma 3.5 we obtain the equation $g=n g_{*}+((n-1)(m-1)) / 2$, where $g_{*}$ denotes the genus of $M / \phi$. If either $n>g$ or $n=g=2$ or 3 , then exactly one of the following possibilities holds:
(i) $n=g=2, g_{*}=0$, and $m=5$.
(ii) $n=g=3, g_{*}=0$, and $m=4$,
(iii) $n=g+1, g_{*}=0$, and $m=3$,
(iv) $n=2 g+1, g_{*}=0$, and $m=2$.

Since $g_{*}=0, \alpha^{\wedge}$ is the trivial knot. By Lemma 3.2, $\left[\alpha^{n}\right]^{\wedge}(=K)$ is a fibred knot.

THEOREM 3.7. Let $L$ be an $n$-periodic link in $S^{3}$ of braid index 3 or 4 . If $L$ has an $n$-periodic minimal braid representation, then $e(\beta) \equiv 0(\bmod n)$ for any minimal braid representation $\beta$ of $L$, where $e(\beta)$ denotes the exponent sum of the braid $\beta$ as a word on the $\sigma_{i}$ 's.

PROOF: If $L$ has an $n$-periodic minimal braid representation, then there is a minimal braid representation $\alpha^{n}$ such that $L$ is ambient isotopic to the closure of $\alpha^{n}$. Since the exponent sum in a minimal braid representation of a link of braid index 3 or 4 is a link invariant [5], $e\left(\alpha^{n}\right)=e(\beta)$ for any minimal braid representation $\beta$ of $L$. But $e\left(\alpha^{n}\right)=n e(\alpha) \equiv 0(\bmod n)$. This completes the proof.

TheOREM 3.8. Let $L$ be an $n$-periodic alternating fibred link in $S^{3}$. If $L$ has an $n$-periodic minimal braid representation, then $w\left(D_{L}\right) \equiv 0(\bmod n)$, where $D_{L}$ is a reduced alternating diagram of $L$ and $w\left(D_{L}\right)$ is the writhe of $D_{L}$.

Proof: Let $L$ be an $n$-periodic alternating fibred link in $S^{3}$ and let $L$ have an $n$-periodic minimal braid representation of the form $\alpha^{n}$. In [8], it is shown that the exponent sum in a minimal braid representation of an alternating fibred link $L$, denoted by $e(L)$, is a link invariant and $e(L)=w\left(D_{L}\right)$, where $D_{L}$ is a reduced alternating diagram of $L$. Thus $w\left(D_{L}\right)=e(L)=e\left(\alpha^{n}\right)=n e(\alpha) \equiv 0(\bmod n)$.

\section{Example 3.9.}

(1) The torus knot $K_{2, q}(q>2)$ is an alternating fibred knot and the exponent sum $e\left(K_{2, q}\right)$ of $K_{2, q}$ is equal to $q$. By Theorem 3.8, $K_{2, q}$ has no 2-periodic minimal braid 
representation. Note that $K_{2, q}(q \geqslant 2)$ has a $d$-periodic minimal braid representation for any divisor $d$ of $q$. The knot $8_{19}$ has periods 2,3 and 4 and $8_{19}=\left[\left(\sigma_{1} \sigma_{2}\right)^{4}\right]^{\wedge}$. Hence it has 2- and 4-periodic minimal braid representation. Also the knot $10_{124}$ has periods 3 and 5 and $10_{124}=\left[\left(\sigma_{1} \sigma_{2}\right)^{5}\right]^{\wedge}$. Hence it has 5 -periodic minimal braid representation. But $e\left(8_{19}\right)=8, e\left(10_{124}\right)=10$. Since 3 is relative prime to 8 and 10 , by Theorem 3.7, $8_{19}$ and $10_{124}$ of braid index 3 have no 3 -periodic minimal braid representations.

(2) 57 out of 117 prime knots of braid index 4 up to 10-crossings in Rolfsen's table [11] are periodic knots with only period 2 except 940 which has another period 3 $[5,7]$ and has a 3-periodic minimal braid representation (see Example 4.5). Since their exponent sums in minimal braid representations [5] are all odd numbers, by Theorem 3.7 , those 57 knots have no periodic minimal braid representations.

\section{PERIODIC LINKS OVER THE SAME FACTOR LINK}

Let $L$ be an oriented link in $S^{3}$ and let $\beta$ be a braid representation of $L$. Then the closed braid $\left[\beta^{n}\right]^{\wedge}$ is clearly an $n$-periodic link in $S^{3}$ whose factor link is ambient isotopic to $L$. Hence we can obtain many periodic links over the same factor link. But, as discussed in section 3 , there are periodic links without any periodic braid representation. In this section we give a construction of periodic links with the same factor link (see [2]). Throughout this section we always assume that links and their diagrams under considerations are polygonal.

DEFINITION 4.1: Let $a, b$ be positive real numbers and let $R=[0, a] \times[0, b]$. Let $a_{i}, b_{i}(1 \leqslant i \leqslant n)$ be the $n$ points on boundaries $\{0\} \times[0, b]$ and $\{a\} \times[0, b]$ of $R$, respectively, where the $y$-coordinate of $a_{i}$ is equal to that of $b_{i}$ for each $i=$ $1,2, \cdots, n$, and $\gamma_{1}, \cdots, \gamma_{n}$ curves in $R . T=\left(R,\left\{\gamma_{1}, \cdots, \gamma_{n}, c_{1}, \cdots, c_{m}\right\}\right)$ is called an (unoriented) tangle diagram of degree $n$ if it satisfies the following conditions;

(1) Each of the points $a_{1}, \cdots, a_{n}, b_{1}, \cdots, b_{n}$ is one of the end points of one of the curves $\gamma_{1}, \cdots, \gamma_{n}$.

(2) $c_{1}, \cdots, c_{m}$ are closed curves contained in the interior of $R$

(3) There is no triple crossing point of the curves.

(4) Every curve has a marking at each crossing point indicating whether the curve under consideration is the over path or the under path at the crossing.

(5) The intersection of the curves $\gamma_{1}, \cdots, \gamma_{n}$ and the boundary of $R$ is equal to $\left\{a_{1}, \cdots, a_{n}, b_{1}, \cdots, b_{n}\right\}$.

If orientations are given to all the curves in $R$, then $T$ is called an oriented tangle diagram of degree $n$. Two tangle diagrams $T$ and $T^{\prime}$ of degree $n$ are said to be 
equivalent if $T$ is ambient isotopic to $T^{\prime}$ keeping the boundary fixed. The closure of a tangle diagram $T$, denoted by $T^{\wedge}$, means the link obtained by identifying $a_{i}$ with $b_{i}$, for each $i=1,2, \cdots, n$. For two tangle diagrams $T$ and $T^{\prime}$ of degree $n$, the product $T T^{\prime}$ is defined by identifying $b_{i}$ of $T$ with $a_{i}^{\prime}$ of $T^{\prime}$ for each $i=1,2, \cdots, n$.

Let $L$ be an oriented link in $S^{3}=\mathbb{R}^{3} \cup\{\infty\}$ and let $D$ be an oriented link diagram of $L$ in the interior of the rectangle $R=[0, a] \times[0, b]:(a, b>0)$ in $\mathbb{R}^{2}$. Let $G_{D}$ be the directed planar graph in the rectangle $R$ with either 2-valent or + or - signed 4-valent vertices obtained from $D$ by assigning to each crossing of $D$ the + or - sign as shown in Figure 4.1.


Figure 4.1

We may assume that all the $y$ coordinates of vertices in $G_{D}$ are distinct real numbers. Throughout this paper the set of directed edges of $G_{D}$ is denoted by $E\left(G_{D}\right)$. The voltage assignment on $G_{D}$ into a group $\Gamma$ is a map $\tau: E\left(G_{D}\right) \rightarrow \Gamma$ such that $\tau\left(e^{-1}\right)=\tau(e)^{-1}$, where $e^{-1}$ means a directed edge from the vertex $v$ to the vertex $u$ for a directed edge $e$ from $u$ to $v$.

For a vertex $v$ in $G_{D}$, the star of $v$, denoted by $\operatorname{star}(v)$, is the subgraph consisting of the vertex $v$ and all the vertices adjacent to it and of all the edges joining those other vertices to $v$.
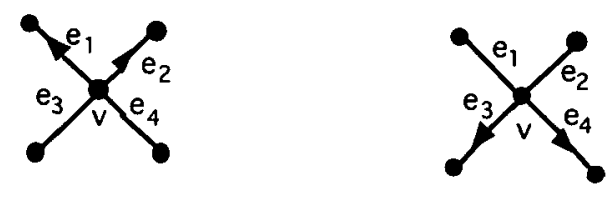

Figure $4.2 \operatorname{star}(v)$

DEFinition 4.2: A voltage assignment $\tau: E\left(G_{D}\right) \rightarrow \mathbb{Z}$ is said to be proper if $\tau(e) \geqslant 0$ for all $e \in E\left(G_{D}\right)$ and for each 4-valent vertex $v$ of $G_{D}$ whose star $\operatorname{star}(v)$ is one of the two types in Figure 4.2, at least one of the upper edges $\left\{e_{1}, e_{2}\right\}$ in $\operatorname{star}(v)$ has zero voltage and at least one of the lower edges $\left\{e_{3}, e_{4}\right\}$ in $\operatorname{star}(v)$ has zero voltage.

For a proper voltage assignment $\tau: E\left(G_{D}\right) \rightarrow \mathbb{Z}$, we construct the oriented tangle $T\left(G_{D}, \tau\right)$ as follows.

First we choose an ordering $e_{1}, e_{2}, \cdots, e_{m}$ of all the edges with non-zero voltages and then for the first directed edge $e_{1}=u_{1} v_{1}$ with $\tau\left(e_{1}\right)=k$, let $u_{1}=\left(x_{1}, y_{1}\right), v_{1}=$ 

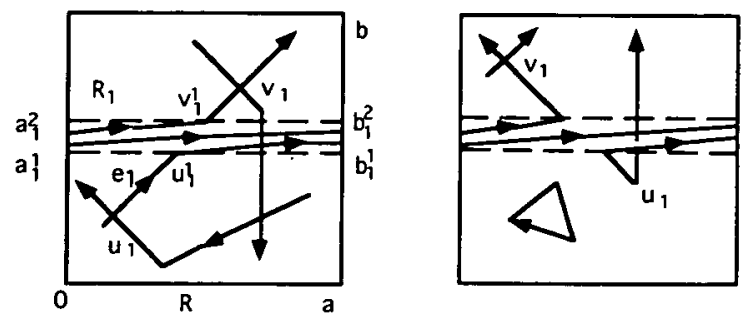

$\tau\left(e_{1}\right)=2$ and $y_{1}<y^{\prime}$
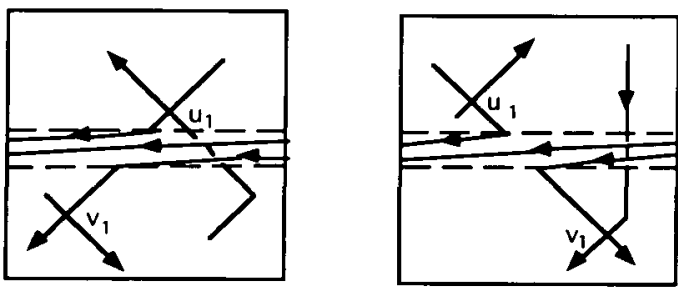

$\tau\left(e_{1}\right)=2$ and $y_{1}>y_{1}^{\prime}$

Figure $4.3 T\left(G_{D}, \tau\right)$

$\left(x_{1}{ }^{\prime}, y_{1}{ }^{\prime}\right)$ in rectangular coordinates. If $y_{1}<y_{1}{ }^{\prime}$, let $R_{1}=\left\{(x, y) \in R \mid y_{1}+\varepsilon_{1} \leqslant y \leqslant\right.$ $\left.y_{1}+\varepsilon_{1}^{\prime}, 0 \leqslant x \leqslant a\right\}$ be the subrectangle in $R$ for sufficiently small $0<\varepsilon_{1}<\varepsilon_{1}^{\prime}$ so that $R_{1}$ is so close to the vertex $u_{1}$ that it can contain no other vertices of $G_{D}$. Note that $e_{1} \cap R_{1}$ is a small line segment $e_{1}^{1}=u_{1}^{1} v_{1}^{1} \subset R_{1}$. Let $a_{1}^{1}, a_{1}^{2}, \cdots, a_{1}^{k}$ be the $k$ points on the line segment $\{0\} \times[0, b] \cap R_{1}$ and let $b_{1}^{1}, b_{1}^{2}, \cdots, b_{1}^{k}$ be the $k$ points on the line segment $\{a\} \times[0, b] \cap R_{1}$ such that the $y$-coordinate of each $b_{1}^{i}$ is equal to that of $a_{1}^{i}$. Deleting the line segment $e_{1}^{1}$, we connect $u_{1}^{1}$ to $b_{1}^{1}, a_{1}^{k}$ to $v_{1}^{1}$, and $a_{1}^{i}$ to $b_{1}^{i+1}, i=1,2, \cdots, k-1$, by the directed straight lines in $R_{1}$ whose directions are compatible with the direction of $e_{1}$ so that the crossings with other edges of $G_{D}$ are all overcrossings (see Figure 4.3). If $y_{1}>y_{1}{ }^{\prime}$, then by letting $R_{1}=\left\{(x, y) \in R \mid y_{1}-\varepsilon_{1}{ }^{\prime} \leqslant y \leqslant y_{1}-\varepsilon_{1}, 0 \leqslant x \leqslant a\right\}$ for sufficiently small $0<\varepsilon_{1}<\varepsilon_{1}^{\prime}$ so that $R_{1}$ is so close to the vertex $u_{1}$ that it can contain no other vertices of $G_{D}$, we can follow the above procedure.

By applying this process to $e_{2}, e_{3}, \cdots, e_{m}$ repeatedly, we obtain the $m$-tangle diagram $T\left(G_{D}, \tau\right) \in \mathbb{T}_{m}$ by deciding the over or under crossing at each + or signed 4-valent vertex in $T\left(G_{D}, \tau\right)$ according to its sign (see Figure 4.1), where $m=\sum_{e \in E\left(G_{D}\right)} \tau(e)$. Since the voltage assignment is proper, the resulting tangle $T\left(G_{D}, \tau\right)$ does not depend on the choice of the ordering of the edges with non zero voltages. It depends on the voltage assignment $\tau$ on $G_{D}$. Obviously the closure of $T\left(G_{D}, \tau\right)$ is ambient isotopic to $L$. 
THEOREM 4.3. Let $L$ be an oriented link in $S^{3}=\mathbb{R}^{3} \cup\{\infty\}$ with an oriented link diagram $D$ in the interior of the rectangle $R=[0, a] \times[0, b](a, b>0)$ in $\mathbb{R}^{2}$. Let $G_{D}$ be the graph of $D$ in $R$ and let $\tau: E\left(G_{D}\right) \rightarrow \mathbb{Z}$ be a proper voltage assignment on $G_{D}$. Then the closure of the self-product $T\left(G_{D}, \tau\right)^{n}(n>1)$ of the $m$-tangle $T\left(G_{D}, \tau\right)$ is an $n$-periodic oriented link in $S^{3}$ whose factor link is ambient isotopic to $L$. Conversely every periodic link in $S^{3}$ can be constructed in this way.

Proof: The first part of the theorem is obvious (see Figure 4.4).

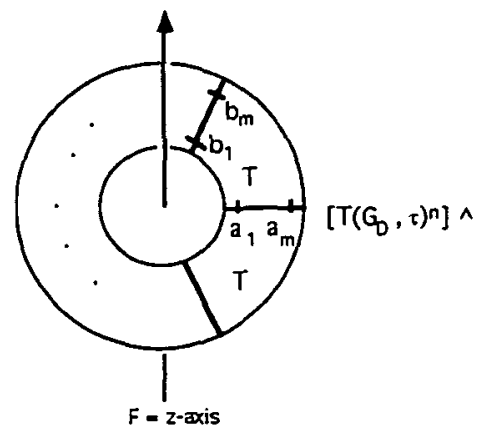

Figure 4.4

Conversely, let $L$ be an oriented $n$-periodic polygonal link in $S^{3}$ whose factor link is $L_{*}$ and let $\phi:\left(S^{3}, L\right) \rightarrow\left(S^{3}, L\right)$ be the periodic homeomorphism. By the positive solution of Smith Conjecture, the set $F$ of fixed points of $\phi$ is unknotted. If we consider $S^{3}$ as $\mathbb{R}^{3} \cup\{\infty\}$, then we may assume that $F$ is the $z$-axis with $\infty$ and $\phi$ is the standard $(2 \pi) / n$-rotation of $\mathbb{R}^{3}$ about the $z$-axis and $\phi(\infty)=\infty$. By taking the regular projection of $L$ into $\mathbb{R}^{2}, L$ is represented by a polygonal diagram $D$ in an annulus in $\mathbb{R}^{2}$, which is divided into $n$-pieces $D_{0}, D_{1}, \cdots, D_{n-1}$ such that $\varphi\left(D_{i}\right)=D_{i+1}, i=0,1, \cdots, n-2, \varphi\left(D_{n-1}\right)=D_{0}$, where $\varphi$ is the restriction of $\phi$ on $\mathbb{R}^{2}$. Let $a_{1}, a_{2}, \cdots, c_{m}$ denote the intersection points of $D_{0}$ with the $x$-axis and let $\varphi\left(a_{i}\right)=b_{i}, i=1,2, \cdots, m$. By connecting $a_{i}$ to $b_{i}$ by polygonal path $c_{i}$ with the orientation induced from $D_{0}, i=1,2, \cdots, m$ (see Figure 4.5), we obtain a polygonal link diagram $D_{*}$ of $L_{*}$. Let $G_{D_{*}}$ be the graph of $D_{*}$. Without loss of generality we may assume that $G_{D}$, is contained in the rectangle $R$. Now define a voltage assignment $\tau: E\left(G_{D_{*}}\right) \rightarrow \mathbb{Z}$ by assigning voltage 1 to the unique directed edge of $c_{i}$ connected to $a_{i}$ for each $i=1,2, \cdots, m$ and voltage 0 to all other directed edges of $G_{D \text {. (see }}$ Figure 4.5). Notice that the voltage assignment $\tau$ is obviously proper.

By applying the construction to the graph $G_{D_{n}}$, we obtain the $m$-tangle $T\left(G_{D_{*}}, \tau\right)$. The closure of the product $T\left(G_{D_{*},}, \tau\right)^{n}$ is an oriented $n$-periodic link which is ambient 

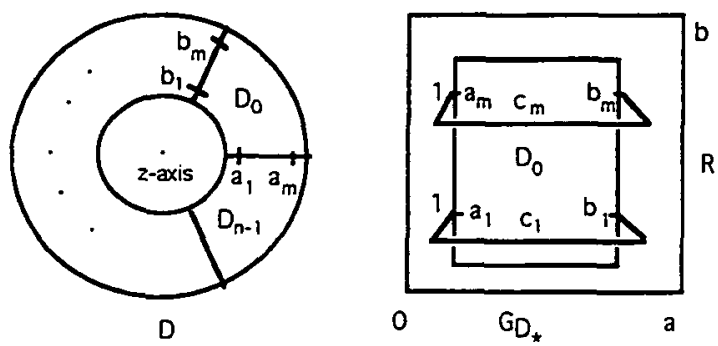

Figure 4.5

isotopic to $L$ since $T\left(G_{D_{*}}, \tau\right)$ is ambient isotopic to $D_{0}$ keeping the boundary fixed. This completes the proof.

The following Corollary 4.4 is an immediate consequence of Theorem 4.3 and Lemma 3.2.

Corollary 4.4 . Let $D$ be an oriented diagram of the trivial knot and let $\tau: E\left(G_{D}\right) \rightarrow \mathbb{Z}$ be a proper voltage assignment. Assume that the tangle $T\left(G_{D}, \tau\right)$ is equivalent to a braid. Then the link $L_{D}^{\tau}=\left[T\left(G_{D}, \tau\right)^{n}\right]^{\wedge}$ is an $n$-periodic fibred link in $S^{3}$ which has an $n$-periodic braid representation.

We conclude by giving an application of the construction.

EXAMPLE 4.5. Let $L$ be the trivial knot and let $D$ be its diagram as shown in Figure 4.6. Let $G_{D}$ be the graph of a diagram $D$ with edges $e_{1}=v_{1} v_{2}, e_{2}=v_{2} v_{3}, e_{3}=$ $v_{3} v_{4}, e_{4}=v_{4} v_{5}, e_{5}=v_{5} v_{3}$, and $e_{6}=v_{3} v_{1}$. Let $\tau_{i}(i=1,2, \cdots, 8)$ be the proper voltage assignments from $E\left(G_{D}\right)$ to $\mathbb{Z}$ defined as shown in the table of Figure 4.6.

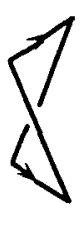

D

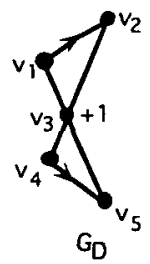

\begin{tabular}{|c|cccccc|}
\hline$i$ & $e_{1}$ & $e_{2}$ & $e_{3}$ & $e_{4}$ & $e_{5}$ & $e_{6}$ \\
\hline 1 & 0 & 1 & 0 & 2 & 0 & 0 \\
2 & 0 & 1 & 2 & 2 & 0 & 0 \\
3 & 0 & 2 & 1 & 2 & 0 & 0 \\
4 & 0 & 2 & 0 & 2 & 0 & 0 \\
5 & 1 & 1 & 2 & 0 & 0 & 0 \\
6 & 0 & 0 & 0 & 1 & 1 & 2 \\
7 & 0 & 1 & 0 & 1 & 1 & 0 \\
8 & 0 & 0 & 0 & 1 & 2 & 0 \\
\hline
\end{tabular}

Figure 4.6

Then we have

$$
\begin{aligned}
& 4_{1}=\left[T\left(G_{D}, \tau_{1}\right)^{2}\right]^{\wedge *}=\left[\left(\sigma_{2} \sigma_{1}^{-1}\right)^{2}\right]^{\wedge}, \quad 6_{2}=\left[T\left(G_{D}, \tau_{2}\right)^{2}\right]^{\wedge}=\left[\left(\sigma_{4}^{-1} \sigma_{3}^{-1} \sigma_{2}^{-1} \sigma_{1}\right)^{2}\right]^{\wedge}, \\
& 7_{6}=\left[T\left(G_{D}, \tau_{3}\right)^{2}\right]^{\wedge}=\left[\left(\sigma_{4}^{-1} \sigma_{3}^{-1} \sigma_{2} \sigma_{1}^{-1}\right)^{2}\right]^{\wedge}, 8_{18}=\left[T\left(G_{D}, \tau_{1}\right)^{4}\right]^{\wedge *}=\left[\left(\sigma_{2} \sigma_{1}^{-1}\right)^{4}\right]^{\wedge},
\end{aligned}
$$




$$
\begin{array}{rlrl}
9_{40} & =\left[T\left(G_{D}, \tau_{4}\right)^{3}\right]^{\wedge}=\left[\left(\sigma_{3}^{-1} \sigma_{2} \sigma_{1}^{-1}\right)^{3}\right]^{\wedge}, & 9_{47} & =\left[T\left(G_{D}, \tau_{5}\right)^{3}\right]^{\wedge *}=\left[\left(\sigma_{3} \sigma_{2} \sigma_{1}^{-1}\right)^{3}\right]^{\wedge}, \\
10_{123} & =\left[T\left(G_{D}, \tau_{1}\right)^{5}\right]^{\wedge *}=\left[\left(\sigma_{2} \sigma_{1}^{-1}\right)^{5}\right]^{\wedge}, & 5_{1}^{2}=\left[T\left(G_{D}, \tau_{5}\right)^{2}\right]^{\wedge}=\left[\left(\sigma_{3}^{-1} \sigma_{2}^{-1} \sigma_{1}\right)^{2}\right]^{\wedge}, \\
6_{3}^{2} & =\left[T\left(G_{D}, \tau_{4}\right)^{2}\right]^{\wedge *}=\left[\left(\sigma_{3} \sigma_{2}^{-1} \sigma_{1}\right)^{2}\right]^{\wedge}, & 6_{2}^{3}=\left[T\left(G_{D}, \tau_{1}\right)^{3}\right]^{\wedge}=\left[\left(\sigma_{2}^{-1} \sigma_{1}\right)^{3}\right]^{\wedge}, \\
9_{35} & =\left[T\left(G_{D}, \tau_{6}\right)^{3}\right]^{\wedge}, 9_{41}=\left[T\left(G_{D}, \tau_{7}\right)^{3}\right]^{\wedge}, & 9_{49}=\left[T\left(G_{D}, \tau_{8}\right)^{3}\right]^{\wedge *},
\end{array}
$$

where $L^{*}$ means the mirror image of the link $L$. Hence we conclude that $4_{1}, 6_{2}, 7_{6}$, $8_{18}, 9_{40}, 9_{47}, 10_{123}, 5_{1}^{2}, 6_{3}^{2}$, and $6_{2}^{3}$ are fibred links which have periodic braid representations. Note that $44_{1}, 7_{6}, 8_{18}, 9_{40}, 9_{47}$, and $10_{123}$ have periodic minimal braid representations. Also $\left[T\left(G_{D}, \tau_{6}\right)^{3}\right]^{\wedge},\left[T\left(G_{D}, \tau_{7}\right)^{3}\right]^{\wedge}$, and $\left[T\left(G_{D}, \tau_{8}\right)^{3}\right]^{\wedge}$ are link diagrams of the 3-periodic knots $9_{35}, 9_{41}$ and $9_{49}$, respectively, invariant under the corresponding periodic homeomorphism (see Figure 4.7).

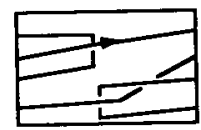

$T\left(G_{D}{ }^{16}\right)$

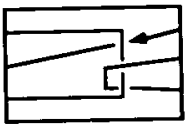

$T\left(G_{D}, \tau 7\right)$



$T\left(G_{D} \tau_{B}\right)$

Figure 4.7

\section{REFERENCES}

[1] J.W. Alexander, 'A lemma on systems of knotted curves', Proc. Nat. Acad. Sci. U.S A. 9 (1923), 93-95.

[2] Y. Bae and C.-Y. Park, 'Alexander polynomials of periodic links', (preprint).

[3] J.S. Birman, Braids, links, and mapping class groups, Ann. of Math. Studies. 82 (Princeton Univ. Press, 1975).

[4] A.L. Edmonds, 'Least area Seifert surfaces and periodic knots', Topology Appl. 18 (1984), 109-113.

[5] V.F.R. Jones, 'Hecke algebra representations of braid groups and link polynomials', Ann. Math. 126 (1987), 335-388.

[6] C.McA. Gordon, R.A. Litherland and K. Murasugi, 'Signature of covering links', Canad. J. Math. 33 (1981), 381-394.

[7] K. Kodama and M. Sakuma, 'Symmetry groups of prime knots up to 10 crossings', in Knots 90 (Osaka 1990) (de Gruyter, Berlin, 1992), pp. 323-340.

[8] K. Murasugi, 'On the braid index of alternating links', Trans. Amer. Math. Soc. 326 (1991), 237-260.

[9] K. Murasugi, 'On periodic knots', Comment. Math. Helv. 46 (1971), 162-174.

[10] K. Murasugi, 'On a certain subgroup of the group of an alternating link', Amer. J. Math. 85 (1963), 544-550.

[11] D. Rolfsen, Knots and links (Publish or Perish Inc, Berkeley, CA, 1976). 


\section{Department of Mathematics \\ College of Natural Sciences \\ Kyungpook National University \\ Taegu 702-701 \\ Republic of Korea}

